\title{
OUTLINE OF LECTURE ON GUNSHOT FRACTURES OF THE JAWS AND THEIR TREATMENT.
}

\author{
By John S. Marshall, M. D., Sc. D., F. A. C. S. \\ Captain U. S. Army, (Retired) Berkeley, Cal.
}

(Read before the Preparedness League of American Dentists, San Francisco, Cal.)

$F^{R}$ RACTURES of the Jaws. By the term fracture is meant a break in the continuity of the bone.

Fractures of the bones of the body are of common occurrence and make up a very considerable portion of the practice of the general surgeon. Inasmuch as the change in the title of our lecture confines us to "Gunshot Fractures of the Jaws" we shall have very little to say about fractures of the jaws as seen in Civil Life.

In peace times fractures of the jaws in our Army, follow very closely the percentages recorded in civil life.

The lower jaw or mandible on account of its more exposed position and its peculiar shape is much more liable to injury from accidents than is the upper part of the face.

Fractures of the Jaws are quite common in our Army even during times of peace. These injuries generally result from blows upon the face from the closed fist; kicks of horse or mule; the impact of some heavy missile propelled with considerable force; the extraction of teeth; tho this is rare; a fall from a horse, bicycle, or a gun-carriage; the passage of a wheel over the face; and gunshot injury in line of duty or from accident, or design, with suicidal intent or otherwise.

Gunshot Fractures, except in time of war are very rare. The army dental surgeon might serve for ten years with- out seeing a single case of this character.

In times of war, however, the circumstances would be very different and it might be his fortune, and probably would be, to see many such cases during the period of his service.

Trench warfare and the increased use of Shrapnel has greatly augmented the number of wounds of the head, face and upper portion of the body, while it has greatly decreased the number of wounds of the trunk and lower limbs. Severe wounds of the head, face and neck are very apt to prove fatal from injury to the brain or the large blood-vessels.

The Face Wounds which escape an immediate termination are usually those made by the jacketed bullet portions of shrapnel or fragments of shell, which tear away the soft tissues and underlying bone, leaving a hideous and ghastly wound.

Injury to the bone from these missiles is often very great, smashing and comminuting it to such an extent that it seems practically impossible to restore the normal contour of the injured part, and yet some of our English, French, German and American confreres have been able, by their superior skill and scientific technic to accomplish results that are well nigh miraculous.

The Oral Surgeon has during this World War come into his own. He is now recognized as an indispensable 
member of the Surgical : Staff of every well organized Civil, and Military Hospital.

Because of the achievements of our confrerers upon the other side of the Atlantic, much, very much, will be expected and required of $u s$. Can we arise to the occasion? Surely we can; and we must.

There is a possibility that this war may go on for another two or three years, and if this be true, there will be great need for skillful oral surgeons and very many of them.

It is your duty to prepare yourselves to meet this emergency when it shall come.

What I shall say to you this evening will be a mere outline of the subject before us. More than this time will not parmit.

The subject matter as laid down in the printed programm2 is so comprehensive that it would require at least three lectures to cover it, and that only in a superficial manner.

Fractures of the Maxillary Bones are classified under two general forms; namely, Simple and Complicated.

A Simple Fracture is one in which there is a single break in the bone, without laceration of the external tissues.

Complicated Fractures include all other conditions associated with a fracture of a bone such as injuries to the external tissues, to vessels, nerves, teeth, a comminuted condition of the bone itself, or any other condition which complicates a simple fracture.

Complicated Fractures may be divided into Multiple, Comminuted and Compound.

Multiple Fractures are those which have more than one break in the continuity of the bones.

Comminuted Fractures are the result of crushing injuries, or gunshot wounds, which crush and splinter the bone into minute fragments.

Compound Fractures are those which have associated with them injuries to the soft tissues, causing exposure of the fractured bone.

The last two forms are those most often seen in Military Surgery and will be the only forms discussed this evening.

The military oral surgeon will, however, be often called upon to treat fractures of the jaws which are not the result of gunshot injuries, consequently every form of fracture from the simplest to the most complex will be presented for treatment.

All gunshot fractures of the jaws belong to, and come within the two last mentioned groups, namely: Compound and Comminuted Fractures.

Fractures of this character are almost invariably infected from the moment of the injury; either from the missile itself, the septic condition of the skin, portions. of which are carried into the deeper tissues of the wound, or the septic condition of the mouth and the accessory sinuses.

In Comminuted Fractures of the Jaws, fragments of the bone and of teeth are of ten carried into the tissues for a considerable distance where they by reason of their septic condition, establish many separate foci of infection.

These conditions greatly complicate the case and render the treatment difficult and hazardous. Another, immediate and often dangerous complication, is hemorrhage and this may call for the ligation of the external carotid artery, if the patient's life is to be saved.

Secondary Hemorrhage is sometimes a serious complication and calls for prompt action for its control.

Sepsis and Hemorrhage are the two most serious complications that must be combatted.

Primary Hemorrhage, that which occurs immediately after the injury, if it does not at once prove fatal is usually treated by the surgeons upon the firing line.

Secondary Hemorrhage usually de- 
velopes in from forty-eight hours to two weeks after the injury has been inflicted.

There is still another serious complication that sometimes developes, namely, gangrene.

This condition is largely due to the smashing effect of the missile upon the soft tissues, which kills them outright; to the interruption of the circulation thru the severing or ligation of important blood-vessels that supply the part; or to infection with the gas producing organism bacillus welchii of gas gangrene, (emphysematous gangrene).

This form of gangrene was little known until the present World War. It is a septic condition induced by the above named micro-organism, acting upon injured, or dead and dying tissues. Because of the fact that gas gangrene is one of the most serious of all wound complications, it may not be out of place at this time to say a few words about the organism that causes the disease.

This organism like that of tetanus (lock-jaw) is an anaerobic bacillus, and grows and flourishes without the presence of oxygen. It is found in the intestinal tract of the higher animals, in garden soil, street dust, sewage, river water, and milk, but it is most abundant in soil.

Its action after entering a wound is to produce necrosis of the soft tissues, followed by liquifaction and putrifaction of the tissue elements, with the evolution of gas.

It is most often found associated with injuries to the bone. Compound and Comminuted Fractures.

Wounds infected with this organism have been very common among the soldiers from the battle-fields of Northern France. This form of infection has given the surgeons at the front, and also at the base hospitals in France, Germany and in England a great deal of trouble and much anxiety.

A little over a year ago, in a communication received from an English
Surgeon, he stated "nearly all of the cases that are sent home from France are in a septic condition, many of them gangrenous, and not a few of these, but many, are due to the presence of the gas bacillus."

Infection with this particular microorganism has been thought by some authorities to be due to its presence in the soil of the land over which the opposing armies have been contending. Northern France, and in fact the whole area of battle-scarred France and Belgium have been for generations subjected to the highest forms of cultivation and fertilization, consequently the soil is filled with many forms of bacteria that are dangerous to the health and the life of man if by chance they gain an entrance to his body, not the least of which are the tetanus bacillus and the gas bacillus.

Tetanus antitoxin is used in large quantities at the dressing stations and the field hospitals as a preventive measure against tetanus, and it is due to this fact that so few cases of this disease have developed during these later months of the war.

For the proper presentation of my subject it will be necessary to slightly change the order of the subsections as laid down in the printed program and add some others. Shock for instance is not mentioned and this is important.

\section{A-Surgical Treatment.}

Shock if present, is the first condition that needs attention. Operation should be deferred, except for hemorrhage, until the patient rallies from the condition of shock.

1.-Shock is a condition of sudden depression of the functions of the whole body, due to powerful impressions upon the system by physical injury or mental emotion.

The symptoms or manifestations, are signs of lowered activity of the cardiac, respiratory, and sensorial functions, and reduction of surface temperature. The depression of the vital forces corre- 
sponds to the severity of the injury and the impression made upon the nervecenters. The tendency is generally towards reaction which may take place in a few minutes or be delayed for several hours or in very severe cases it may never be established and the case terminate fatally.

The prognosis of shock is considered unfavorable if reaction does not set in within eighteen to twenty-four hours, or if the body temperature falls below $96^{\circ}$ F. A surgical operation under such conditions is absolutely contra-indicated.

Other unfavorable symptoms are loss of power of swallowing, loss of sensation in the conjunctiva, persistent vomiting, and relaxation of the sphincter muscles, inducing involuntary evacuation of the bladder and of the bowels.

The Treatment of Shock. In mild cases of shock very little in the line of treatment is required beyond that of keeping the patient warm and quiet. The application of artificial heat by means of hot-water bags, hot-sand bags, or hot bricks, so protected as not to burn the patient, is of great value in restoring the equilibrium of the circulation and the nervous forces. Stimulants and other drugs are not indicated, except in severe cases, as they have a tendency, by increasing the blood-pressure and heart action, to reestablish or increase hemorrhage.

Always place a patient suffering from shock, in the prone position, upon the back and when the heart action is much - depressed, the foot of the bed should be elevated so as to bring the head lowest, that all unnecessary labor upon the heart may be removed and the least possible amount of energy expended in forcing the blood to the exhausted vital nerve-centers.

If the shock is due to loss of blood it may be necessary to employ normal salt solution by subcutaneous injection or the transfusion of blood by the veines.

2.-Sepsis and Antisepsis. As a rule, all battle injuries are septic from the moment of their infliction, as already stated.

Such injuries or wounds, however, should be immediately protected from further infection by the employment of the contents of the first aid package found in every soldier's kit. All handling of the wound as in attempts io cleanse it only increase the dangers from further infection.

Wounds of the face and jaws, by reason of these parts as a rule remaining uncovered, are not so often infected by portions of clothing, as are wounds made upon portions of the covered body.

In any event the wound must first be exposed, but not touched by the hands, or washed, as these efforts at cleansing only increase the dangers from infection.

The first aid package is sterile and contains two bandages and a small concontainer of Tincture of Iodine for disinfection.

The long bandage with the compress sewed upon it, at the middle, is for protecting a wound of the face, trunk, or extremeties; the other is what is known as a handkerchief bandage and may be employed as a sling or for covering a wound of the head.

After a wound has been uncovered the tincture of iodine is poured into it and the bandage immediately applied. This gives the necessary protection until the wounded individual can be removed to the dressing station.

At the dressing station the case receives such other attention as the conditions demand and he receives a dose of tetanus antitoxin. As soon as it is safe to move him he is forwarded by Ambulance to the Field Hospital, where a careful and painstaking examination is made and recorded, and further treatment instituted against the development of tetanus.

Only emergency operations are performed at the Field Hospitals. 
As rapidly as the conditions of the patients will permit and the circumstances of transport allow, they are forwarded to the Base Hospitals for further treatment and necessary operations.

The Oral Surgeons are usually stationed at the Base Hospitals, and consequently rarely see the cases of Fracture of the Jaws, until several days after the infliction of the injury and suppuration has generally been well established.

Very few cases of Compound fracture of the jaw in Civil practice escape suppuration, and practically none in Military practice.

Gunshot Fractures of the Jaws usually involve a more or less extensive destruction and loss of bone and teeth. Occasionally only the teeth and a portion of the supporting alveolar process will be carried away; while in another case not only the teeth and a portion of the alveolar process, but a considerable section of the body of the bone is comminuted and lost.

Injuries which carry away large sections of the maxillary bones are very serious and as a rule cause great facial deformity.

Inasmuch as this lecture is supposed to deal with those fractures of the jaws which are caused by gun-shot injuries or in other words war injuries, we shall not attempt to deal with those less serious fractures of the jaws due to accidents not associated with the use of fire-arms and which make up the bulk of jaw injuries as seen in civil practice.

Gunshot Fractures of the Jaws and Bones of the Face make up a very considerable part of the wounds found assembled in the European Base Hospitals and for this reason the services of trained oral and dental surgeon have been in great demand: The former for his superior skill and knowledge in treating mouth and jaw injuries, and the latter for his no less great skill and technic in the construction of splints and artificial appliances to take the place of those structures which have been destroyed and carried away.

The cooperation of the general surgeon, the oral specialist and the dentist, have thru this team-work accomplished many things that seem little less than miraculous if we may believe the reports that have been published from time to time in our periodical literature. Some of us might be inclined to doubt these statements, if it were not for the oral testimony of some of our confrerers who have been eye-witnesses of these marvels.

Symptoms of Gunshot Fractures. The symptoms of this class of injuries are so plain that "he that runs may read." An open wound of the face, and deformity in symmetry and contour, are sufficient to indicate the nature of the injury. No two cases are ever likely to present the same characteristics, or require identical treatment. Hence there must be a never ending interest in the subject, and a variety of studies presented, which will tax the skill and inventive resources of the various numbers of the staff to their full limit.

The opportunities for growth and development along these special lines has never been surpassed in the history of the world.

It is of course necessary to examine the wound that a proper diagnosis may be made, but let the examination be as free from traumatisms as possible. Let there be no unnecessary probing or manipulation of the tissues as this increases the liability to further infection.

All loose pieces of bone, or fragments of clothing or of the missile causing the injury, -and this may be portions of a bullet, of Shrapnel, of shell, of wood, or portions of accounterments of the victim or of a comrade-should be removed.

Pieces of bone, however, having periosteal attachments should be allowed to remain, as the chances are that they will form the nucleus for the regeneration of bone. 
A fragment of bone with a vital attachment to the periosteum is superior to a bone graft even under the most favorable circumstances. Much harm is often occasioned by the misdirected, over-zealous efforts of some young enthusiastic surgeon, along the line of cleaning out the wound by the removal of fragments of bone.

Much serious deformity might be avoided if small fragments of attached bone were allowed to remain.

It is surprising to see how readily these little pieces will unite and form a considerable plate of bone. This is particularly noticed in the external wall of the frontal sinus, the maxillary sinus and of the bones of the nose.

My advice along this line is never remove any fragment of bone that has a vital connection with the periosteum.

These fragments if depressed as in the regions just mentioned may be manipulated into place with proper instruments and moulded into position, and thus reduce the liability to a more or less serious deformity.

\section{SETTING FRACTURES OF THE JAWS.}

As was said on a previous page, "no two fractures caused by gunshot injury ever present the same characteristics or require identical treatment" consequently each case must be treated according to its individual requirements.

For instance, an injury involving the loss of the crowns of several teeth and a portion of the alveolar process, with a simple fracture of the body of the jaw, would require a much more simple method of treatment than would an injury which carried away a large section of the jaw upon one or both sides. The former might be treated, if it occurred in the anterior portion of the jaw, by an external splint and a four-tailed bandage; while that involving a loss of bone upon one side of the jaw might be very satisfactorily treated by wiring the jaws together in a normal occlusion, and later constructing a bridge of porcelain and one of the precious metals to replace the lost tissues. An injury involving the loss of a portion of the body of the jaw upon both sides would not only be a serious wound, but a very difficult one to treat particularly so if associated with the lower jaw. Hemorrhage might prove a serious complication, and greatly retard any efforts made to place the jaw in a relatively normal position. This may be accomplished temporarily by the aid of an external splint and bandages. Swelling of the soft parts would in all probability be very considerable, rendering it well-nigh impossible to operate in any form for several days. In these cases salivation is very great, and the taking of food out of the question without the aid of the stomach tube.

The efforts of the surgeon in these cases is primarily addressed to relieving the pain, to reducing the swelling, and combating the infection. As soon as the swelling has been reduced and the infection overcome, operative procedures may be undertaken.

Treatment. The treatment of a case of this character would naturally require team-work suich as we spoke of a few moments ago; work that would call for the highest degree of technical skill upon the part of all parties engaged.

We take it for granted that there is only one method of restoring a jaw to usefulness that has been so seriously injured as the one under discussion, and that would be by bone grafting.

An operation of this character, presenting as it would, so many difficulties and possible discouragements, would involve an immense amount of preliminary preparations before the surgical treatment could be undertaken. First, it would be necessary to take impressions of both jaws. From these, models should be made with Plaster of Paris. There would be considerable difficulty in securing suitable impressions of the lower jaw for in all probability it would 
be necessary to take it in sections and then after models were made, to reconstruct these using the model of the upper jaw as a guide for the proper occlusion of the teeth.

Upon these reconstructed models it would be necessary to construct an interdental splint that would hold the anterior and posterior fragments of the jaw in their relatively normal position, during such time as would be required for the grafted bone to unite with the anterior and posterior fragments.

The construction and insertion of the interdental splint should not be delayed beyond the arrest of suppuration and the healing of the traumatisms within the mouth, as it not infrequently happens that undesirable adhesions are formed which may require operation to overcome.

The treatment by bone grafting would require many weeks and perhaps months to accomplish a successful result and during all of this time there would be the constant liability to loss of the graft from necrosis of infection or of both.

An interdental splint such as would be required in a case of this character would have to be so constructed that it could be kept clean by irrigation. This can be easily accomplished by drilling numerous holes in the splint opposite the individual teeth.

(Various splints were. exhibited that had been used in practical cases).

The form of splint required for a case like : the one under discussion should cover all the teeth in both jaws. It would also be necessary to mould an external splint to the lower jaw extending from angle to angle as an added support to the jaw.

The head should now be shaved, the interdental splint placed in position, the external splint for the lower jaw applied, the mouth firmly closed by upward pressure upon the external splint and the jaws so held together by adhesive strips passed under the chin and over the crown of the head.

This forms an immovable appliance that can be kept clean and that will hold the parts in their normal position, thus preventing undesirable adhesions and will maintain the fragments of the lower jaw in admirable position for the operation of bone grafting and during the process of union between the graft and fragments of the jaw.

No grafting of bone should be undertaken while there is any sign of suppuration within the mouth or elsewhere for reasons that are obvious.

Grafting of bone would have to be done thru external incisions and these should be so placed as to prevent an unsightly scar. Care must be taken not to enter the oral cavity as infection would be fatal to the graft. The bone for the graft is best taken from the patient himself.

There are two methods of making bone grafts in the mandible. The most reliable method is to cut a section of bone from the jaw. itself long enough to fill the gap, leaving the fragment attached to its periosteum and over lying tissues, sliding it into place and retaining it in position either with silver wire structures or bone dowels. This preserves the blood-supply of the graft and greatly increases the chances of a successful operation. It is not essential that the bulk (the gross bulk) of the graft be as great as the portion of the jaw that has been lost, for a very small piece, if it fills the gap, will form its environment and the stimulation of use, soon attain a size commensurate with the normal requirements of the jaw.

The other method is to transplant a section of bone from a rib or from the anterior portion of the tibia. Be sure at all times to leave the periosteal covering of the bone intact.

The transplanted bone must be of such length as will fill the gap and it 
must be secured in position by means of the wire suture or the bone dowels.

In this operation there is a fair chance that a sufficient re-establishment of the blood circulation will take place between the graft and the tissues into which it is transplanted, and that union will take place between the graft and the fragments of the jaw.

Dr. Murphy was of the opinion that the transplanted bone, as such, never united with the living bone of the part, but that it simply acted as a bridge, framework or scaffolding for the regeneration or formation of new bone, and that finally this bridge or scaffolding was removed by a process of resorption as the new bone was formed. It was also his opinion that the periosteum was not so essential to bone regeneration as some authorities had maintained, but that the medullary tissue was the active agent in the formation of new bone.

From several X-ray pictures shown me by Dr. Murphy of cases which he had under treatment at the Mercy Hospital, I am inclined to believe this is the true explanation of the manner in which nature performs this marvelous service.

Clinical experience also seems to point in this direction, as for instance in certain compound fractures of the mandible with loss of considerable sections of the bone by necrosis, it not infrequently happens that this lost tissue is replaced by new bone. And I have also seen the same phenomenon take place in gunshot fractures of the jaw, where quite extensive portions of the bone have been completely carried away. The filling up of the gap seemingly by growth of new bone from the ends of the fracture.

I have known of one case, a Fillipino boy 16 years of age, in which a gap of a full inch was filled in, in this way, and five years afterwards defied detection, except as shown by the absence of certain belonging to this region.

Of course all surgery of this character, must be performed under the very strictest aseptic methods, from beginning to end.

Your essayist has had some little experience in the treatment of gunshot fractures of the Jaws, and also with bone grafting, hence speaks from knowledge of the difficulties to be overcome.

It was my privilege to make my first bone grafting operation upon a lower jaw for a woman (in January 1887) who had eight years previously lost a large section of the right half of the jaw in the removal of a malignant growth. This operation of bone grafting was performed at the Mercy Hospital in Chicago where the late lamented. Dr. John B. Murphy, so recently made such a brilliant record in bonegraft surgery.

\section{REPAIRING SOFT TISSUES.}

The terrible deformities of the face and jaws occasioned by the modern weapons of warfare has stimulated the ingenuity and artistic taste and ability of the modern surgeon to such a degree that it is now well-nigh possible to reconstruct the features of the individual to such an extent as to almost make a new face, and sometimes even to improve upon the original itself.

At the beginning of this World War Plastic Surgery had not been very highly developed and there were very few surgeons capable of handling these cases with any great degree of efficiency. The necessity, however, of doing something to relieve the great depression of mind of these poor fellows occasioned by these ghastly deformities was the chief stimulus to the intellect and heart of the surgeon. Out of this necessity has developed the most wonderful skill and technic in plastic surgery that the world has ever seen or is even likely to see again.

The extent and severity of the injuries to the face and jaws is often very great.

It not infrequently happens that a considerable portion of the cheeks, lips or nose may be badly torn or completely carried away leaving an unsightly, re- 
volting deformity that can only be remedied by a masterful plastic operation.

One of the chief difficulties that the surgeon has to encounter is to obtain the tissue with which to close the gap. Ordinary skin grafting is not always suitable as for instance in restoring a nose, in building a new lip or filling a large perforation in a cheek. In these cases tissue en masse must be transplanted. Such tissue must come from a location near the seat of the injury, as during the healing in of the transplanted tissue it must maintain a vital circulatory connection at its base, otherwise the tissue will die and slough out.

Dr. Carrel's experiments-now interrupted by his service in France-gave great promise of his being able to keep alive certain tissues for a more or less indefinite period, and of actually transplanting them from one organism to another. Whether this will ever be accomplished in a practical manner we do not know, but the Good Book tells us that if we "have faith as a grain of mustard seed we must say to yon mountain be ye removed into the sea and it will be done." What appears today to be a miracle is tomorrow a common place phenomenon. Let us therefore have faith.

Twenty-five to fifty years ago great dependence was placed upon mechanical appliances as substitutes for lost portions of the face and mouth as for instance artificial noses, artificial palates, artificial jaws, etc.

Today the surgeon restores the nose by a plastic operation. He also closes a perforation in the hard palate caused by specific disease or a gunshot injury by the same means; while loss of bone tissue in the jaw or the mandible is replaced by a bone graft, which after a few months is so completely restored that it is difficult for the uninitiated to detect it, or to believe that such a serious injury had ever been inflicted, or an operation of such magnitude had ever been performed.

We are only just upon the threshold of a great and marvelous new era in surgery, an era that will be as wonderful and as full of possibilities as that which followed the discoveries of anesthesia and of antiseptics. What a supreme and exalted privilege it is, and will be, to live in such an age, and add our mite, no matter how small it may be to the furtherance of such a glorious epoch.

Do we realize our high privilege?

Are we as practitioners of dental and oral surgery ready and willing to assume the responsibilities of this high privilege and take the place in the great profession of the Healing Art, that circumstances has opened to us? I trust and believe that we are and now that the time for action is upon us, let us take our place and fulfill our obligations to the best of our ability. Knowing that "of him who has received much, much will be required." 